\title{
Effect of reaction protocol on the nature and size of iron oxide nano particles obtained through solventless synthesis using iron(II)acetate: structural, magnetic and morphological studies
}

\author{
A. Dey ${ }^{1} \cdot$ M. Zubko ${ }^{2,3} \cdot$ J. Kusz ${ }^{4} \cdot$ V. Raghavendra Reddy $^{5} \cdot$ A. Bhattacharjee ${ }^{1}$ (I)
}

Received: 27 August 2019 / Accepted: 9 December 2019 / Published online: 11 January 2020

(c) Springer Nature Switzerland AG 2020

\begin{abstract}
Magnetite and hematite nanoparticles ( $20 \mathrm{~nm}$ size) have been synthesized by solventless thermal decomposition of iron(II)acetate in air under different reaction protocols. The structure of the decomposed materials is investigated by FT-IR and powder XRD, while the morphology of the particles formed was studied by SEM/TEM. The magnetic phases of the materials are quantitatively identified by ${ }^{57} \mathrm{Fe}$ Mössbauer spectroscopy. EDX was used for elemental analysis. The results obtained from FT-IR, XRD and Mössbauer studies explicitly establish that the materials obtained are iron oxides (magnetite and hematite). The study shows a reaction time- and temperature-dependent conversion of magnetite to hematite. The underlying solid state reactions have been discussed. The present study comes across that a single organo-iron precursor can provide different pure phases of iron oxide nanoparticles depending on the reaction protocol.
\end{abstract}

Keywords Iron oxides · Nanoparticles · Solventless synthesis · Protocol dependence · Physical characterization

\section{Introduction}

Among the materials ubiquitous in nature, the sixteen types of iron oxide-oxyhydroxides are important as these display a wide range of physical properties [1]. For this variety of physical properties, iron oxides draw great interest in multiple scientific research areas for various application potentials, e.g., optoelectronics, magnetic storage, anticorrosion, imaging, pigments, gas sensors. Among the iron oxides, magnetite $\mathrm{Fe}_{3} \mathrm{O}_{4}\left(\mathrm{Fe}^{3+}\left[\mathrm{Fe}^{3+} \mathrm{Fe}^{2+}\right] \mathrm{O}_{4}\right)$ is a common magnetic oxide having cubic inverse spinel structure with oxygen forming an fcc structure and Fe in the interstitial tetrahedral and octahedral sites. The magnetite nanoparticles have applications in ultrahigh density magnetic storage media, tracking, imaging, biological labeling detection and ferrofluid [2-5]. On the other hand, hematite
$\left(\mathrm{a}-\mathrm{Fe}_{2} \mathrm{O}_{3}\right)$ is a nontoxic, compatible to environment and stable magnetic iron oxide with rhombohedral structure. This material has a wide application potential in the magnetic devices, catalysis, pigments, gas sensors, photoanodes, batteries, etc. [6-11]. Magnetic properties observed in magnetite and hematite are very interesting and are widely discussed in the literature [12-14]. In magnetite at $\sim 123 \mathrm{~K}$, a charge-ordering transition (Verwey transition) occurs when an ordering of $\mathrm{Fe}^{3+}$ and $\mathrm{Fe}^{2+}$ ions within the octahedral sites occurs and a drop in magnetization at the transition temperature is the signature of magnetite $[13,14]$. On the other hand, at $\sim 263 \mathrm{~K}$, hematite exhibits a weak ferromagnetic to antiferromagnetic transition (Morin transition) owing to rearrangement of spins to antiparallel fashion along the rhombohedral (111) axis from a canted spin arrangement with respect to the basal (111) plane

A. Bhattacharjee, ashis.bhattacharjee@visva-bharati.ac.in | 'Department of Physics, Visva-Bharati University, Santiniketan, India. ${ }^{2}$ Institute of Materials Science, University of Silesia, Chorzow, Poland. ${ }^{3}$ Department of Physics, University of Hradec Králové, Hradec Králové, Czech Republic. ${ }^{4}$ Institute of Physics, University of Silesia, Katowice, Poland. ${ }^{5}$ UGC-DAE Consortium for Scientific Research, Indore, India. 
[15-17]. These magnetic phases of iron oxide along with subphases are very accurately detected by the application of Mössbauer spectroscopy [16, 18].

The magnetic and other physical properties of the iron oxide nanoparticles strongly depend on their shape, size and size-distribution, and two factors-size and surface anisotropy, control the magnetic property of these iron oxide nanoparticles $[19,20]$. There are several methods for preparing iron oxide nanoparticles, e.g., solventless thermal decomposition of iron-containing compounds [21], electrochemical deposition [22], co-precipitation [23], ultrasound irradiation [24], solvothermal method [25], microemulsion [26] and hydrothermal method [27]. Out of these methods, solventless thermal decomposition of iron-containing molecular materials is the most easy and practical technique for synthesis of iron oxide nanoparticles as this method neither requires any special instrument nor involves complicated reaction steps, and, moreover, this method provides a good control over the homogeneity, composition, purity, phase and microstructure of the decomposed products [14, 16, 20,28]. Selection of a proper organo-iron molecular precursor with comparatively low decomposition temperature and a rational reaction protocol may result the desired magnetic iron oxide nanoparticles via solventless thermal decomposition route. The reaction mechanism involved as well as the energy required to initiate the decomposition reaction can be obtained by the reaction kinetic study $[29,30]$. In continuation of our earlier attempts $[14,16]$ to prepare iron oxide nanoparticles, presently we report the synthesis of pure magnetite and hematite nanoparticles (size $\sim 20 \mathrm{~nm}$ ) using iron(II)acetate as the sole precursor under different reaction protocols. The materials synthesized were characterized by different physical techniques like FT-IR, powder XRD and ${ }^{57} \mathrm{Fe}$ Mössbauer spectroscopy where the morphology and compositional analysis were studied by SEM/TEM and EDX, respectively. The observations made are discussed in the present paper.

\section{Experimental}

Iron(II)acetate $\left[\mathrm{Fe}\left(\mathrm{C}_{2} \mathrm{H}_{3} \mathrm{O}_{2}\right)_{2}\right]$, melting point $190-200{ }^{\circ} \mathrm{C}$, was obtained from Sigma-Aldrich and was used for thermal decomposition as a precursor without any further treatment. Thermal decomposition profiles of iron(II) acetate in air, oxygen and nitrogen atmospheres (see Fig. 1) were obtained with a thermogravimetric analyzer (STA 449 F3 Jupiter of Netzsch, Germany). UHP (99.999\%) nitrogen and oxygen gases were used as purge gases to create reaction atmosphere, whereas UHP (99.999\%) nitrogen was used as the protective gas in the instrument. From the observed TG profiles, it is

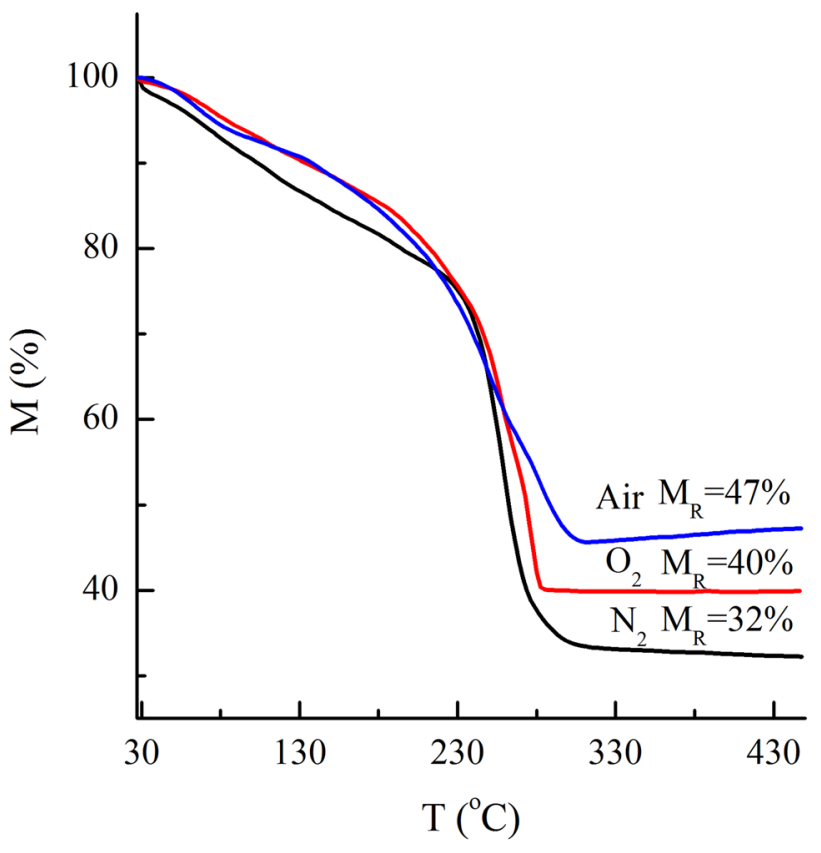

Fig. 1 Thermogravimetry profiles of iron(II)acetate obtained at $3^{\circ} \mathrm{C} /$ min heating rate in $\mathrm{N}_{2}, \mathrm{O}_{2}$ and air atmospheres

clear that the decomposition process becomes complete in the $290-310^{\circ} \mathrm{C}$ range depending on the reaction atmosphere. However, the recorded residual mass $\left(M_{R}\right)$ is highest in the air atmosphere. Thus, the thermal synthesis was carried out by placing certain amount of the iron(II)acetate precursor into an indigenous furnace in air atmosphere at constant temperature. The temperature and duration of reaction were varied as: (a) $1 / 2 \mathrm{~h}$ at $400{ }^{\circ} \mathrm{C}$, (b) $1 \mathrm{~h}$ at $400{ }^{\circ} \mathrm{C}$, (c) $1 \mathrm{~h}$ at $500{ }^{\circ} \mathrm{C}$ and (d) $1 \mathrm{~h}$ at $600{ }^{\circ} \mathrm{C}$ under ambient atmosphere. After the reaction, the samples were cooled down inside the furnace and were collected at room temperature. These thermally synthesized materials are hereafter termed as FA1, FA2, FA3 and FA4 accordingly. These materials were then used for different physical characterization studies.

The synthesized materials were characterized by FT-IR, powder XRD, SEM, EDX, TEM and Mössbauer spectroscopy. FT-IR study was done with a PerkinElmer made (Analytic 10.4.1) spectrometer, whereas the powder XRD study was carried out with PANalytical's Empyrean powder diffractometer equipped with PIXcell ${ }^{3 \mathrm{D}}$ detector and a Cu- $\mathrm{K}_{\mathrm{a}}$ radiation source. Scanning electron microscope (SEM) observations were made with a JEOL JSM-6480 instrument with accelerating voltage of $20 \mathrm{kV}$ equipped with the energy dispersive X-ray spectroscopy (EDX) detector from IXRF. Transmission electron microscopy (TEM) images were recorded using a JEOL JEM 3010 instrument with $300 \mathrm{kV}$ accelerating voltage equipped with $2 \mathrm{k} \times 2 \mathrm{k}$ Orius $^{\mathrm{TM}} 833 \mathrm{SC} 200 \mathrm{D}$ Gatan CCD camera. ${ }^{57} \mathrm{Fe}$ 
Mössbauer spectra were recorded using a conventional constant-acceleration spectrometer with a ${ }^{57} \mathrm{Co}$ Mössbauer source.

\section{Results and discussion}

\subsection{FT-IR study}

FT-IR spectroscopy in the range $4000-400 \mathrm{~cm}^{-1}$ was employed in order to identify the decomposed materials. Figure 2 shows the FT-IR spectrum obtained for FA1, FA2 and $F A 3$ in a reduced range of wave number. The major observed bands at $691,634,541,478 \mathrm{~cm}^{-1}$ for FA1 and at $692,634,527,477 \mathrm{~cm}^{-1}$ for FA2 are due to $\mathrm{Fe}-\mathrm{O}$ bonding observed in magnetite [31-33], whereas bands at $459,435 \mathrm{~cm}^{-1}$ for FA2, $520,434 \mathrm{~cm}^{-1}$ for FA3 and at 519 , $431 \mathrm{~cm}^{-1}$ can be assigned to $\mathrm{Fe}-\mathrm{O}$ in hematite $[34,35]$. These results clearly indicate that FA 1 is purely magnetite; FA2 is a mixture of magnetite and hematite, whereas $F A 3$ is purely hematite.

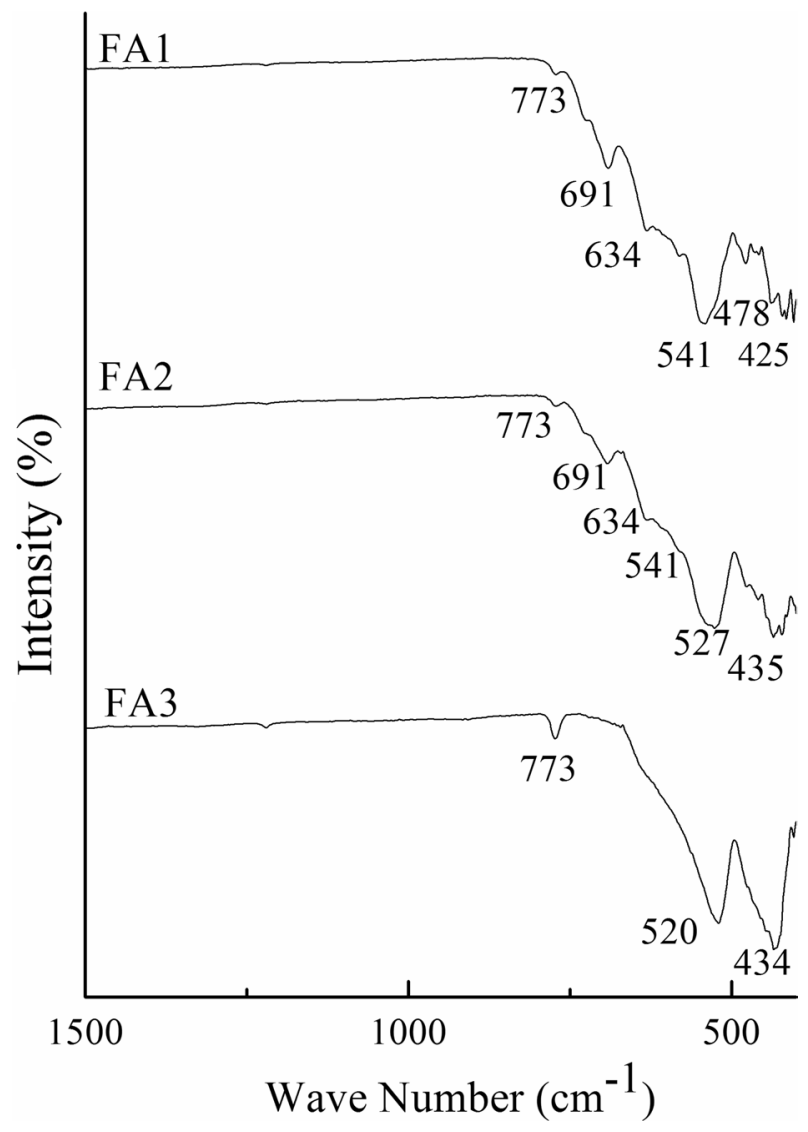

Fig. 2 FT-IR spectra of FA1, FA2, and FA3 - the thermally decomposed materials of iron(II)acetate under different conditions

\subsection{Structural study}

The Rietveld refinement of the XRD patterns of the thermally synthesized materials FA1, FA2, FA3 and FA4 is shown in Fig. 3 where the dot represents the observed data and the line corresponds to the calculated pattern. The curve at the bottom represents the difference between the observed and calculated patterns. The vertical bars show the Bragg positions. A close agreement between the observed and calculated XRD pattern of FA1 establishes that FA 1 is purely magnetite (JCPDF no. 00-065-0731), whereas the XRD pattern of FA2 could be well-fitted to a mixture of magnetite (55\%) (JCPDF no. 00-065-0731) and hematite (45\%) (JCPDF No. 00-0240072). Similarly, the XRD pattern of FA3 clearly exhibits that all of its diffraction peaks correspond to hematite (JCPDF no. 00-024-0072). The powder XRD pattern of FA4 also corresponds to $100 \%$ hematite. The diffraction peaks observed for FA1 are indexed for pure magnetite and for FA3 and FA4 those peaks are of hematite phases, whereas those for FA2 can easily be indexed as the mixture of magnetite and hematite. Thus, heating of the precursor at $400{ }^{\circ} \mathrm{C}$ for $1 / 2 \mathrm{~h}$ produces pure magnetite, whereas heating of the precursor at $400{ }^{\circ} \mathrm{C}$ for further $1 / 2 \mathrm{~h}$ results into the partial conversion of magnetite to hematite. Heating of the precursor for $1 \mathrm{~h}$ at $500{ }^{\circ} \mathrm{C}$ (i.e., $100{ }^{\circ} \mathrm{C}$ above the reaction temperature as in case of FA1) results into the complete conversion of magnetite to hematite. Further increase in temperature (i.e., $600^{\circ} \mathrm{C}$ ) did not bring any change in the composition of the material. The estimated lattice parameters for FA1, FA2, FA3 and FA4 are enlisted in Table 1. The mean crystallite size $(D)$ of these materials is calculated by the Fullprof program based on the full pattern analysis and are compared in Table 1. From Table 1, magnetite crystallites are having size of $\sim 19 \mathrm{~nm}$ in FA1, whereas that of hematite particles are $\sim 24 \mathrm{~nm}$ in FA3 and $\sim 51 \mathrm{~nm}$ in FA4. The thermal decomposition of iron(II)acetate leads to nano-sized magnetite and hematite particles, and this method is a size-controllable technique.

The inter-planer spacing $(d)$ values have been calculated for different (hkl) plane using the strong diffraction peaks for FA1 and FA3 which are found to vary with the crystal plane orientation. In case of FA1 for (220), (311) and (440) planes, the $d$ values are 2.967, 2.532 and 1.485 $\AA$, whereas for (012), (104), (110), (024) and (116) planes of FA3, the $d$ values are $3.684,2.700,2.519,1.841$ and $1.697 \AA$. This orientation dependence of the lattice spacing indicates the presence of strain in these crystalline materials, which might be originated owing to the synthesis conditions. The value strain in these two iron oxide nanoparticles, estimated using Stokes-Wilson equation [36], lies in the range of $7.81 \times 10^{-3}-3.91 \times 10^{-3}$ for FA1 and 
Fig. 3 Powder XRD pattern of FA1, FA2, FA3 and FA4-the thermally decomposed materials of iron(II)acetate under different conditions

$7.17 \times 10^{-3}-3.30 \times 10^{-3}$ for FA3. The strain generated is also related to the dislocation developed in the crystals.

\subsection{Mössbauer study}

Mössbauer spectroscopy is employed to study magnetic materials and in particular to identify quantitatively the multiple magnetic structures in the material [37]. Mössbauer spectra provide different components reflecting each magnetic phase/structure in the mixture of magnetic materials based on their chemical spin state. Thus, the analysis of ${ }^{57} \mathrm{Fe}$ Mössbauer spectral data of an iron-based compound can identify the different ironbased magnetic components present in it and provides quantitative information on the different iron sites [38]. Presently, ${ }^{57} \mathrm{Fe}$ Mössbauer spectroscopy was carried out for FA1, FA2, FA3 and FA4 samples at room temperature to confirm the results obtained through FT-IR and XRD studies. The analyzed spectra of FA1, FA2, FA3 and FA4 are presented in Fig. 4. The Mössbauer spectra were fitted to Lorentzian lines with isomer shift $(\delta)$ with respect to a-iron, quadrupole interaction $\left(\Delta E_{\mathrm{Q}}\right)$, hyperfine field $\left(B_{\mathrm{hf}}\right)$ and the line width as the adjustable fit parameters. The fitted spectra for all the materials consist of two sextets (say, S1 and S2) which is the indication of long-range magnetic order in these materials. Thus, these materials have only magnetic components at room temperature. The fit parameters are presented in Table 2 along with the population of different iron sites in these compounds. The population of iron in different spin sites of magnetite and hematite phases in each of the decomposed materials was estimated from the resonance line area assuming the same the Mössbauer probability of magnetite and hematite.

The Mössbauer spectrum for FA1 is decomposed into two subspectra S1 and S2 where the resonant line of S2 presents significant broadening in comparison to $\mathrm{S} 1$. While fitting, all the fit parameters were set free. Considering the estimated hyperfine field values available in the literature $[18,32]$, the sextets S1 and S2 with hyperfine field $50.4 \mathrm{~T}$ (isomer shift $=0.32 \mathrm{~mm} / \mathrm{s}$ ) and $48.6 \mathrm{~T}$ (isomer shift $=0.32 \mathrm{~mm} / \mathrm{s}$ ) are associated with iron in the tetrahedral and octahedral coordination sites in the magnetite structure, respectively. The population ratio of iron in the tetrahedral and octahedral sites obtained from the subspectral area is quite close to that of ideal ratio (1:2). However, the fitted isomer shift values obtained for the two sextets are close instead of being different as expected [39]. These values are experimentally correct as far as the fitting procedure is adopted. Though not clear at this
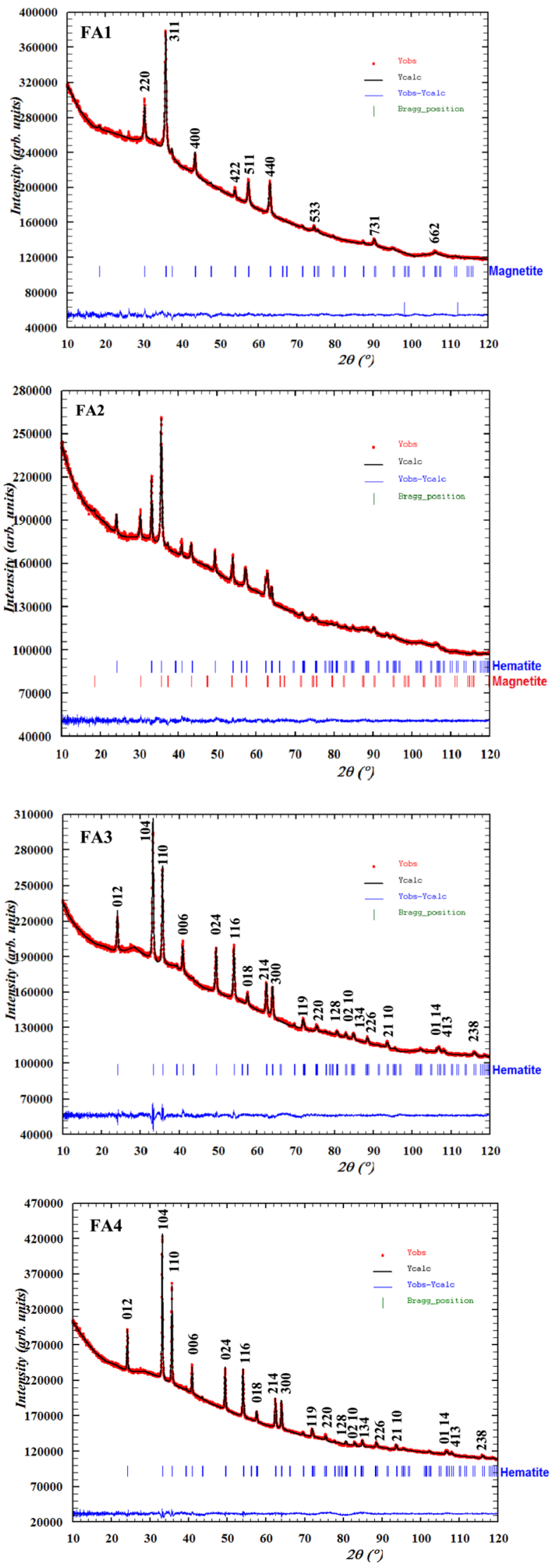
Table 1 Results obtained on the analysis of the powder XRD data obtained for the decomposed materials

\begin{tabular}{lllllll}
\hline Sample & \multicolumn{2}{l}{ Lattice parameters } & & Assignment & Population (\%) & Value of $D(\mathrm{~nm})$ \\
\cline { 2 - 4 } & $a(\AA)$ & $c(\AA)$ & $V\left(\AA^{3}\right)$ & & \\
\hline FA1 & $8.3448(5)$ & & $581.09(7)$ & Magnetite $\left(\mathrm{Fe}_{3} \mathrm{O}_{4}\right)$ & 100 & $18.6(1)$ \\
FA2 & $8.3541(5)$ & - & $583.05(6)$ & Magnetite $\left(\mathrm{Fe}_{3} \mathrm{O}_{4}\right)$ & 54.9 & $18.9(1)$ \\
& $5.0391(3)$ & $13.7655(9)$ & $302.71(3)$ & Hematite $\left(\mathrm{a}-\mathrm{Fe}_{2} \mathrm{O}_{3}\right)$ & 45.1 & $30.8(1)$ \\
FA3 & $5.0384(2)$ & $13.7612(7)$ & $302.54(2)$ & Hematite $\left(\mathrm{a}-\mathrm{Fe}_{2} \mathrm{O}_{3}\right)$ & 100 & $24.3(1)$ \\
FA4 & $5.0376(1)$ & $13.7542(4)$ & $302.29(1)$ & Hematite $\left(\mathrm{a}-\mathrm{Fe}_{2} \mathrm{O}_{3}\right)$ & 100 & $50.9(1)$ \\
\hline
\end{tabular}

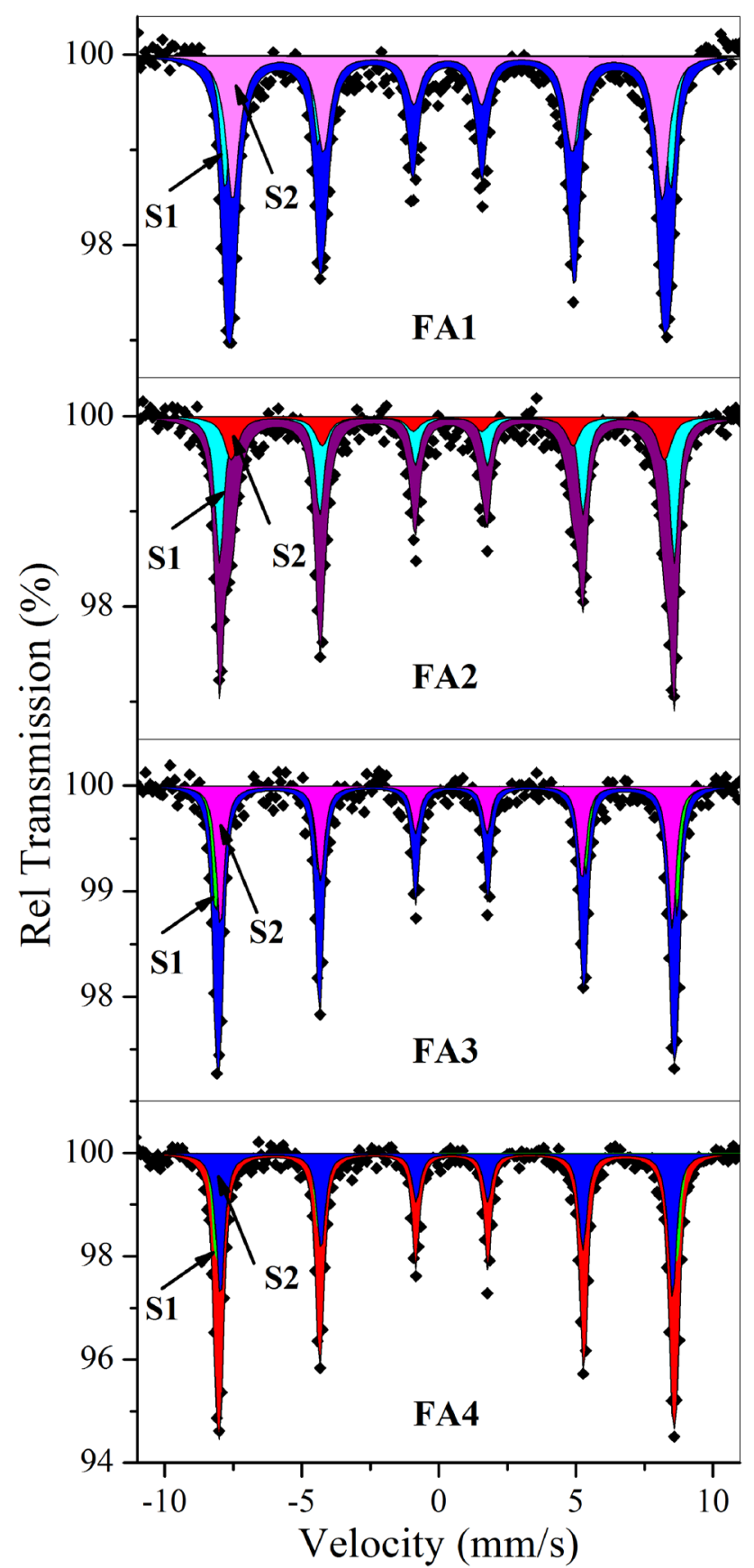

Fig. $4{ }^{57} \mathrm{Fe}$ Mössbauer spectra of FA1, FA2, FA3 and FA4-the thermally decomposed materials of iron(II)acetate under different conditions moment, the observed isomer shift values may be due to incomplete resolution of the experimentally observed spectrum and also related with the small particle size of FA1 sample. It is reported that the small size of magnetite particles shows temperature-dependent motional narrowing to a degree dependent on the mean particle size significantly affecting the hyperfine parameters [40]. Further Mössbauer study at lower temperatures and magnetic field may provide further insights in this regard.

On the other hand, in case of FA2, the sextet $\mathrm{S} 1$ can be assigned to iron in magnetite, whereas the sextet S2 represents iron in hematite phase $[16,18,41]$. The population ratio of these two sites is $\sim 2: 3$. Thus, heating of the precursor at $400{ }^{\circ} \mathrm{C}$ for $1 / 2 \mathrm{~h}$ results into magnetite. On further heating of FA 1 at $400^{\circ} \mathrm{C}$ for additional $1 / 2 \mathrm{~h}$, the iron in magnetite phase at the $\mathrm{S} 1$ site in FA 1 is partially converted to the iron in hematite in FA2, whereas the iron in magnetite phase at the $\mathrm{S} 2$ site in FA1 is fully converted to the iron in hematite in FA2. In FA3, both sextets represent the hematite phase of iron and the population ratio of these two sites is $\sim 2: 3$. From this result, it is clear that on heating FA2 at $500{ }^{\circ} \mathrm{C}$ for $1 \mathrm{~h}$, the iron in magnetite phase at the $\mathrm{S} 1$ site in $\mathrm{FA} 2$ is fully converted to the iron in hematite phase in FA3, whereas the iron in hematite phase at the S2 site in FA2 does not suffer any further change. In FA4, both sextets represent the hematite phase of iron and the population ratio of the two sites is $\sim 3: 7$. Thus, it appears that on heating $F A 3$ at $600^{\circ} \mathrm{C}$ for $1 \mathrm{~h}$, there is no change in the magnetic phase of the iron representing $\mathrm{S} 1$ and $\mathrm{S} 2$ sextets, but the iron in the hematite phase at the $\mathrm{S} 1$ site in FA3 is depoplated increasing the population of the iron in the hematite phase at the S2 site in FA4. From XRD results, the synthesized materials are of single phase except FA2 where magnetite and hematite are present in $\sim$ 1:0.8 ratio. The Mössbauer study of these materials adequately supplements the results obtained from XRD studies. Thus, the Mössbauer spectroscopic study of the materials obtained on thermal decomposition of iron(II)acetate under various reaction conditions represents a reaction temperature and time-controlled formation of magnetite from iron(II)acetate followed by conversion of magnetite to hematite. On the other hand, while comparing the presently observed results on the Mössbauer and XRD studies, it is evident 
Table 2 Hyperfine parameters estimated from the ${ }^{57} \mathrm{Fe}$ Mössbauer spectra recorded at room temperature for the materials obtained on thermal decomposition of iron(II)acetate under different reaction conditions

\begin{tabular}{lllrlll}
\hline Sample & FWHM & $\delta(\mathrm{mm} / \mathrm{s})$ & $\Delta E_{\mathrm{Q}}(\mathrm{mm} / \mathrm{s})$ & $B_{\mathrm{hf}}($ Tesla) & Population (\%) & Assignment \\
\hline FA1 & $0.42 \pm 0.01$ & $0.32 \pm 0.01$ & $0.02 \pm 0.01$ & $50.4 \pm 0.1$ & 32.3 & Magnetite (S1), tetrahedral \\
& $0.59 \pm 0.02$ & $0.32 \pm 0.01$ & $-0.01 \pm 0.01$ & $48.6 \pm 0.1$ & 67.7 & Magnetite (S2), octahedral \\
FA2 & $0.39 \pm 0.01$ & $0.37 \pm 0.01$ & $-0.16 \pm 0.01$ & $51.5 \pm 0.1$ & 58.3 & Hematite (S1) \\
& $0.60 \pm 0.08$ & $0.32 \pm 0.01$ & $0.01 \pm 0.01$ & $49.0 \pm 0.1$ & 41.7 & Magnetite (S2) \\
FA3 & $0.31 \pm 0.01$ & $0.38 \pm 0.01$ & $-0.21 \pm 0.01$ & $52.2 \pm 0.1$ & 41.8 & Hematite (S2) \\
& $0.36 \pm 0.08$ & $0.36 \pm 0.01$ & $-0.18 \pm 0.01$ & $51.1 \pm 0.1$ & 58.2 & Hematite (S1) \\
FA4 & $0.28 \pm 0.01$ & $0.37 \pm 0.01$ & $-0.18 \pm 0.01$ & $52.2 \pm 0.1$ & 31.9 & Hematite (S2) \\
& $0.36 \pm 0.08$ & $0.38 \pm 0.01$ & $-0.20 \pm 0.01$ & $51.1 \pm 0.1$ & 68.1 & Hematite (S1)
\end{tabular}

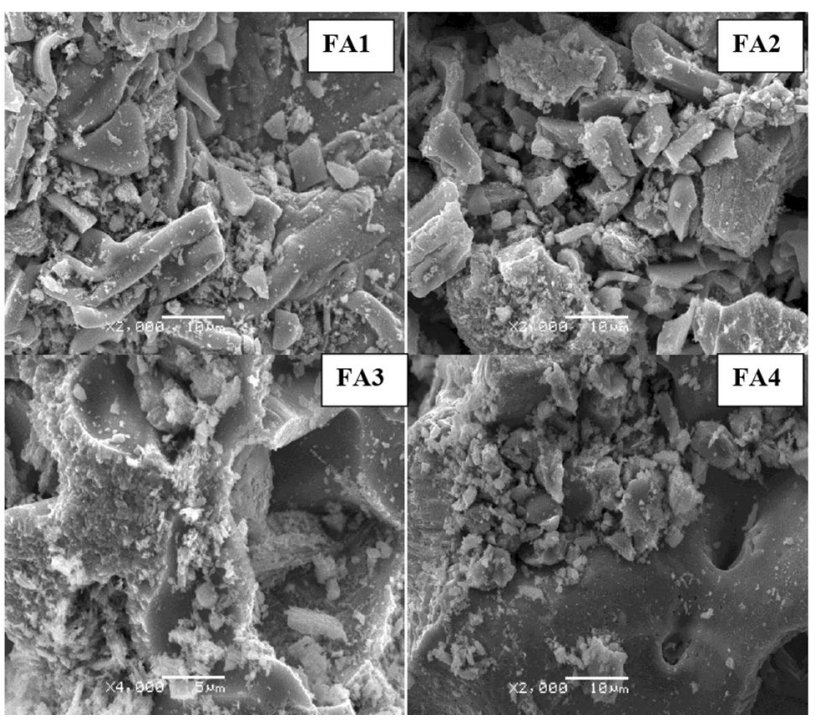

Fig. 5 Selected SEM images of FA1, FA2, FA3 and FA4-the thermally decomposed materials of iron(II)acetate under different conditions

that Mössbauer linewidth increases with decreasing particle size. It is well known that magnetic characteristics of the nanomaterials are strongly affected by the particle size. When particle size becomes very small, thermal energy over the magnetic moment ordering originates the superparamagnetic relaxation phenomenon due to which broadening of the Mössbauer line width occurs [42-45].

\subsection{SEM, TEM and EDX studies}

Morphology of the synthesized iron oxide particles (FA1-FA4) were studied with SEM and TEM, whereas EDX was utilized for elemental analysis. Figure 5 exhibits the selected SEM of FA1, FA2, FA3 and FA4. The SEM images of these materials present the collection of assorted particles. However, the SEM images of FA3 and FA4 are quite different from those of FA1 and FA2. Surface of FA3 appears with large potholes which again contains many small particles of various size and shape, whereas the surface of FA4 contains small potholes and smooth surface also. Figure 6 exhibits the selected TEM of FA1, FA2, FA3 and FA4. The TEM images of FA1, FA2 and FA3 at low magnification show that the particles are agglomerated. However, under high magnification, for FA 1 most of the magnetite particles are found to of irregular shape and a few having hexagonal shape (indicated by arrow). More number of hexagonal nanoparticles are observed in the TEM image of FA2. In case of FA3, the particles are of irregular shape and size. Interestingly, it is found that there are some tiny particles grown on the surface of the large particles (shown by arrow). TEM image of FA4 confirms that the size of particle is larger than that of FA3, which supplements the observation made by $X R D$ that with increase in the reaction temperature and time, the size of the nanoparticle grows. Certainly, the non-uniformity in size and shape observed in these synthesized iron oxide nanoparticles is affected by the nucleation process [46]. Careful observation of the TEM images of FA1, FA2 and FA 3 clearly shows some regions made up of further nano-crystalline phases, shape and lattice plane orientations. Application of high resolution transmission electron microscopy (HRTEM) will help to look into this aspect further. Figure 7 shows the particle size histogram obtained from the TEM image analysis of FA 1 and FA3. From this figure it can be observed that the maximum number of magnetite (FA1) and hematite (FA2) particles obtained are around $27.5 \mathrm{~nm}$ and $42.5 \mathrm{~nm}$, respectively.

Energy dispersive X-ray (EDX) spectroscopy was used to identify the elements present in the samples synthesized. Figure 8 shows some representative EDX spectra of FA1, FA2 and FA3. EDX was done at different spot areas of the specimen samples. The analysis of EDX spectrum revealed the presence of $\mathrm{Fe}$ and $\mathrm{O}$ with no other detectable element. Nearly identical spectra were obtained for different spot areas for each specimen sample which indicate the homogeneity of the elemental composition of the iron oxide nanoparticles synthesized. 

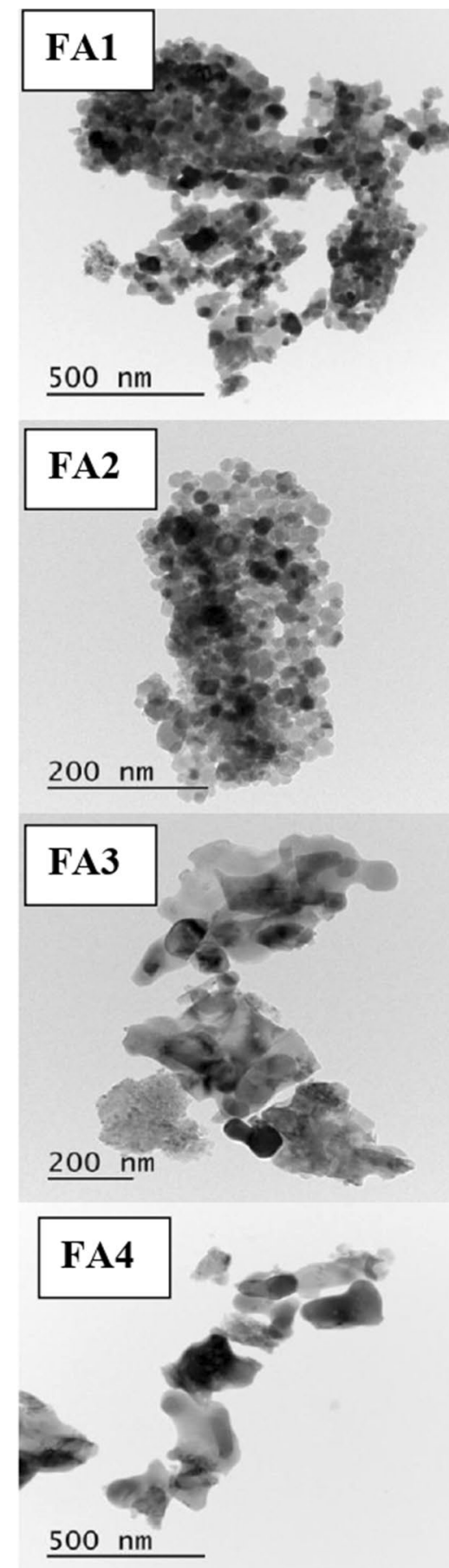

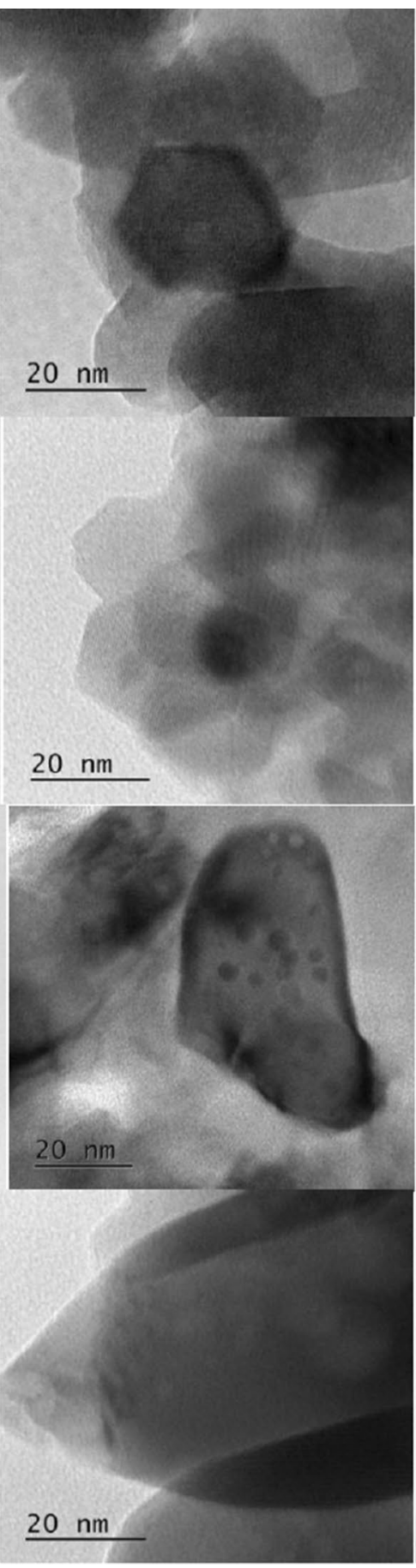

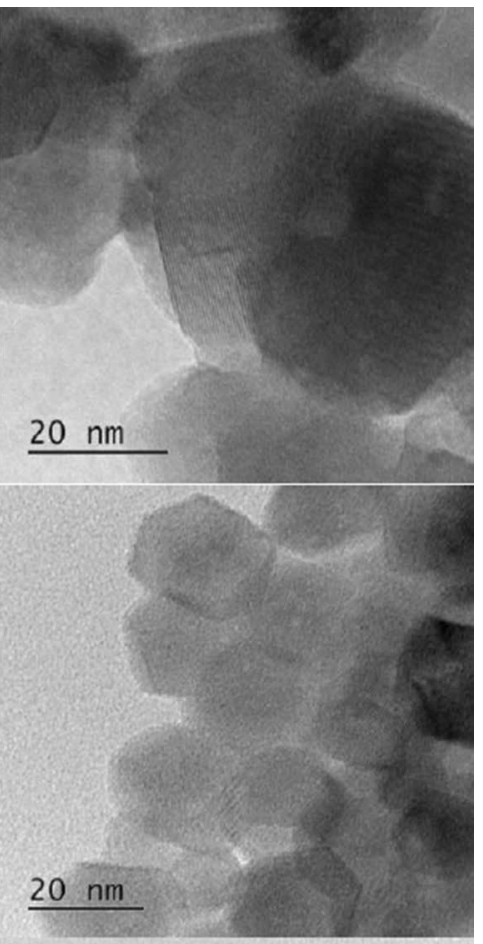
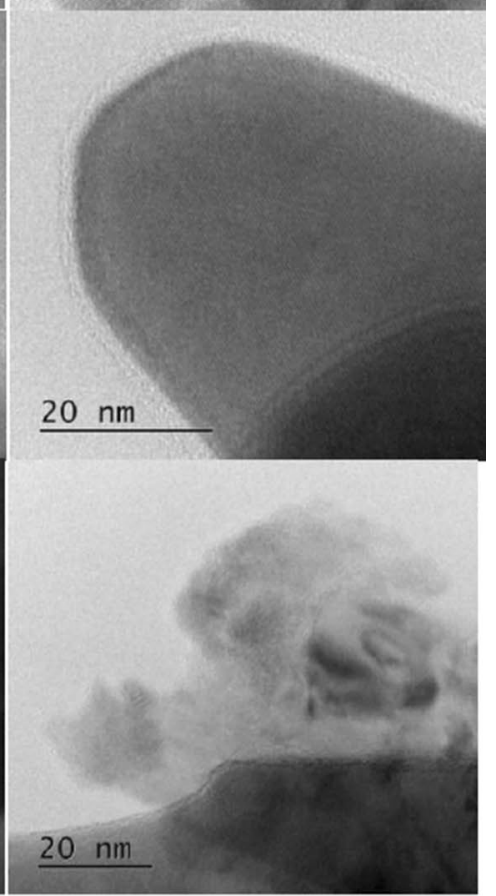

Fig. 6 Selected TEM images of FA1, FA2, FA3 and FA4-the thermally decomposed materials of iron(II)acetate under different conditions

\subsection{Solid state reaction}

Taking the residual mass observed from the TG profile and characterization results, it is understood that on heating from room temperature in air, iron(II)acetate first decomposes to magnetite when kept at $400{ }^{\circ} \mathrm{C}$ for $1 / 2 \mathrm{~h}$ which starts to convert to hematite when kept at the same temperature for further $1 / 2 \mathrm{~h}$. When iron(II)acetate is kept at $500{ }^{\circ} \mathrm{C}$ for $1 \mathrm{~h}$, magnetite is fully converted to hematite. Further rise in reaction temperature did not bring any structural change in the nature of the decomposed material. Considering the compositional analysis of the decomposed materials obtained from XRD studies and the amount of iron concentration in the constituents seen 


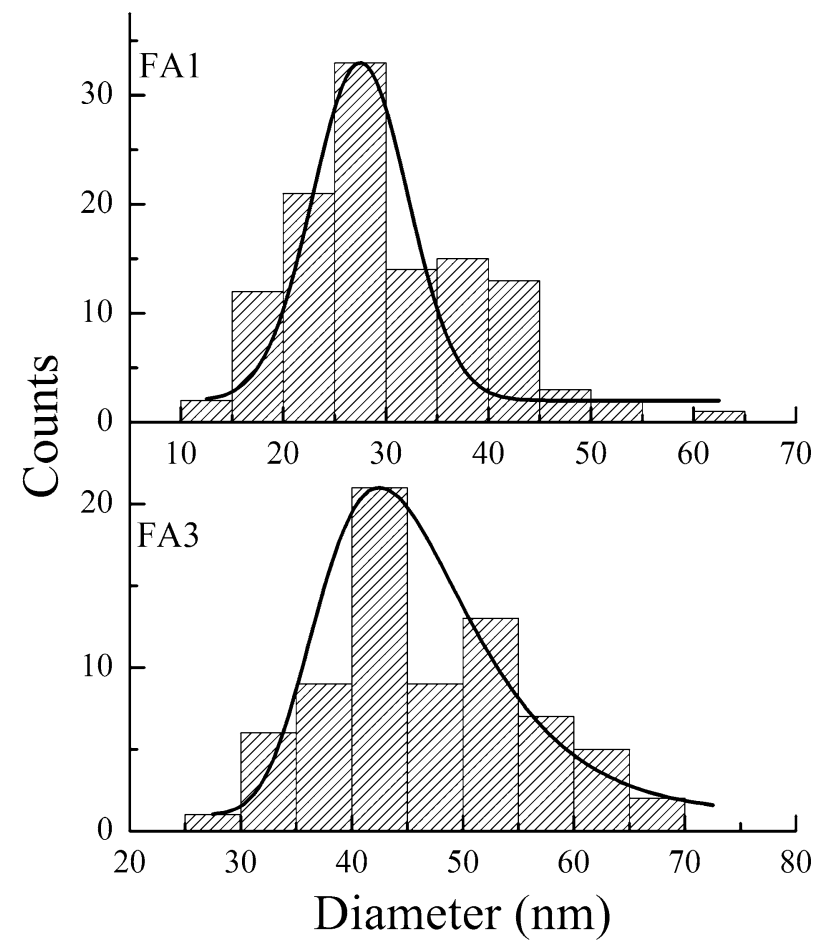

Fig. 7 Histogram of nanoparticles in FA1 and FA2 - the thermally decomposed materials of iron(II)acetate under different conditions

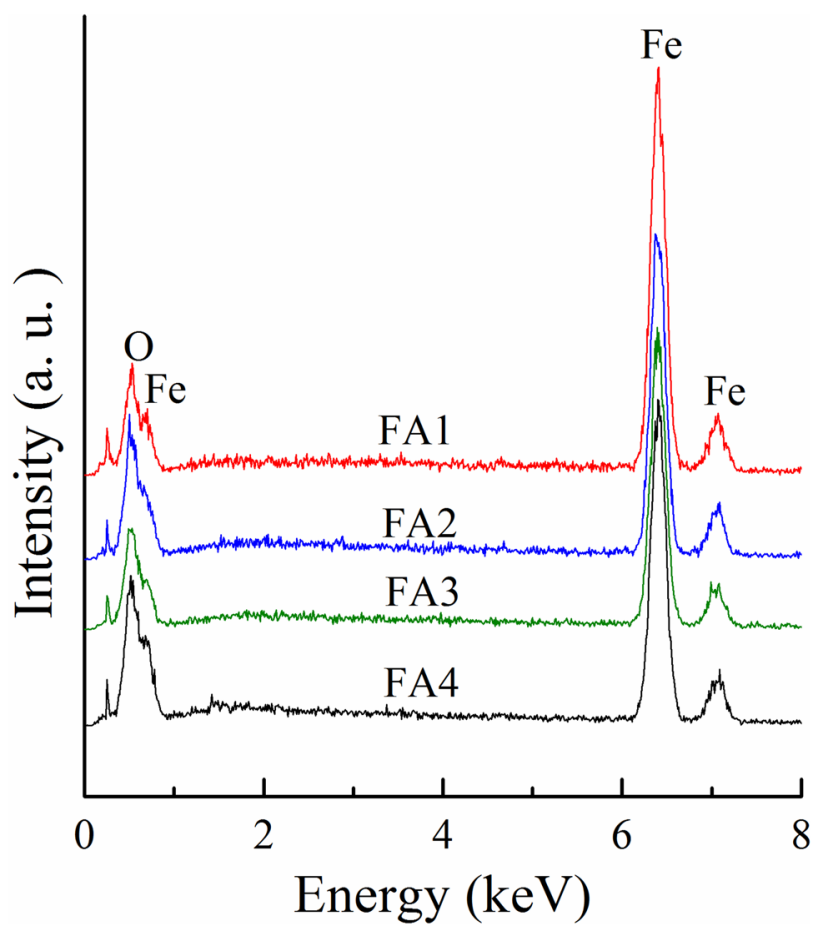

Fig. 8 EDX spectra of FA1, FA2, FA3 and FA4-the thermally decomposed materials of iron(II)acetate under different conditions through Mössbauer spectroscopy, the thermal decomposition reaction of iron(II)acetate in air may be assumed as:

(a) At $400{ }^{\circ} \mathrm{C}$ after $1 / 2 \mathrm{~h}: 3 \mathrm{C}_{4} \mathrm{H}_{6} \mathrm{FeO}_{4}+4 \mathrm{O}_{2}(\mathrm{~g})=\mathrm{Fe}_{3} \mathrm{O}_{4}+8 \mathrm{CO}$ (g) $+4 \mathrm{CO}_{2}(\mathrm{~g})+9 \mathrm{H}_{2}(\mathrm{~g})$

(b) At $400{ }^{\circ} \mathrm{C}$ after $1 \mathrm{~h}: 34 \mathrm{Fe}_{3} \mathrm{O}_{4}+3 \mathrm{O}_{2}$ $(\mathrm{g})=22 \mathrm{Fe}_{3} \mathrm{O}_{4}+18 \mathrm{a}-\mathrm{Fe}_{2} \mathrm{O}_{3}$

(c) At $500{ }^{\circ} \mathrm{C} 1 \mathrm{~h}: 4 \mathrm{Fe}_{3} \mathrm{O}_{4}+4 a-\mathrm{Fe}_{2} \mathrm{O}_{3}+\mathrm{O}_{2}(\mathrm{~g})=10 \mathrm{a}-\mathrm{Fe}_{2} \mathrm{O}_{3}$

' $\mathrm{g}$ ' and ' $\mathrm{s}$ ' denoting gaseous and solid substances, respectively. The calculated residual mass $\left(M_{R}\right)$ values for reaction (a), (b) and (c) are $~ 44 \%, 45 \%$ and $39 \%$, respectively, which are close to the experimentally observed value. A reaction similar to (c) can be assumed for the reaction at $600^{\circ} \mathrm{C}$ for $1 \mathrm{~h}$ duration as the decomposed material is purely hematite as obtained in (c). According to Jubb and Allen [47] magnetite thermally transforms to maghemite $\left(\gamma-\mathrm{Fe}_{2} \mathrm{O}_{3}\right)$ above $200^{\circ} \mathrm{C}$, while above $400^{\circ} \mathrm{C}$ maghemite transform to hematite. On the other hand, Lyubutin et al. [18] observed that on thermal reduction process of hematite, magnetite nanoparticles could be prepared. In the present study, we have observed that during thermal decomposition of iron(II)acetate pure magnetite was formed on oxidation followed by formation of pure hematite through a mixed state of magnetite and hematite.

\section{Conclusion}

Iron(II)acetate has been decomposed under four different reaction conditions by varying reaction temperature and duration of heating in air. The decomposed materials (FA1, FA2, FA3, FA4) were physically characterized by FT-IR, powder XRD, Mössbauer spectroscopy, SEM, TEM and EDX. It has been found that heating of iron(II)acetate at $400^{\circ} \mathrm{C}$ for $1 / 2 \mathrm{~h}$ results in magnetite nanoparticles. An increase in the duration results in the partial conversion of magnetite into hematite. However, increase in the reaction temperature leads to the complete conversion of iron(II)acetate into hematite nanoparticles. Further increase in the reaction temperature leads to the significant increase in the hematite nanoparticles. Guided by the thermal decomposition profile, the possible thermal reactions are proposed supplemented by the quantitative results obtained from the powder XRD and Mössbauer studies. The study shows the reaction time- and temperature-dependent conversion of iron(II)acetate to magnetite and hematite nanoparticles.

Reaction kinetics should control the size, shape and yield of the iron oxide nanoparticles synthesized through solventless thermal decomposition process. In this regard, we are trying to explore the effect of the reaction atmosphere and heating rate as the controlling parameters of the synthesis. Thus, the present study establishes that a 
single organo-iron precursor can provide different pure phases of iron oxide nanoparticles depending on the reaction protocol. This technique can be employed for other metal oxide nanoparticles which are getting our attention too.

Acknowledgements Author AD is thankful to DST-INSPIRE, Govt of India for providing a fellowship and also acknowledges UGC-DAE CSR, Indore, India, for providing financial assistance to carry out the Mössbauer spectroscopic measurements. Authors express thanks to Prof. S. Koner of Department of Chemistry, Jadavpur University, Kolkata, for providing the FT-IR data. Financial support for the thermogravimetry analyzer (STA 449 F3 Jupiter) from the Department of Science and Technology (DST), Govt. of India through a Grant (Ref. No. SR/FIST/PSI-157/2010) to the Department of Physics, Visva-Bharati, Santiniketan is gratefully acknowledged.

\section{Compliance with ethical standards}

Conflict of interest The authors declare that they have no competing interests.

\section{References}

1. Cornell RM, Schwertmann U (1996) The iron oxides: structure, properties, reactions, occurrences and uses. VCH Publishers, Weinheim

2. Zeng H, Li J, Liu JP et al (2002) Exchange-coupled nanocomposite magnets by nanoparticle self-assembly. Nature 420:395-398

3. Nam J-M, Thaxton CS, Mirkin CA (2003) Nanoparticle-based biobar codes for the ultrasensitive detection of proteins. Science 301:1884-1886

4. Raj KMR (1990) Commercial applications of ferrofluids. J Magn Magn Mater 85:233-245

5. Walter $D$ (2006) Characterization of synthetic hydrous hematite pigments. Thermochim Acta 445:195-199

6. Mor GK, Prakasam HE, Varghese OK et al (2007) Vertically oriented $\mathrm{Ti}-\mathrm{Fe}-\mathrm{O}$ nanotube array films: toward a useful material architecture for solar spectrum water photoelectrolysis. Nano Lett 7:2356-2364

7. El-Sheikh SM, Harraz FA, Abdel-Halim KS (2009) Catalytic performance of nanostructured iron oxides synthesized by thermal decomposition technique. J Alloys Compd 487:716-723

8. Salazar-Alvarez G, Qin J, Sepelak V et al (2008) Cubic versus spherical magnetic nanoparticles: the role of surface anisotropy. J Am Chem Soc 130:13234-13239

9. Tseng WJ, Lin R-D (2014) $\mathrm{BiFeO}_{3} / \mathrm{a}-\mathrm{Fe}_{2} \mathrm{O}_{3}$ core/shell composite particles for fast and selective removal of methyl orange dye in water. J Colloid Interface Sci 428:95-100

10. Cesar I, Kay A, Gonzalez Martinez JA, Grätzel M (2006) Translucent thin film $\mathrm{Fe}_{2} \mathrm{O}_{3}$ photoanodes for efficient water splitting by sunlight: nanostructure-directing effect of Si-doping. J Am Chem Soc 128:4582-4583

11. Jain G, Balasubramanian M, Xu JJ (2006) Structural studies of lithium intercalation in a nanocrystalline $\mathrm{a}-\mathrm{Fe}_{2} \mathrm{O}_{3}$ compound. Chem Mater 18:423-434

12. Verwey EJW (1939) Electronic conduction of magnetite $\left(\mathrm{Fe}_{3} \mathrm{O}_{4}\right)$ and its transition point at low temperatures. Nature 144:327-328

13. Piekarz P, Parlinski K, Oleś AM (2007) Origin of the Verwey transition in magnetite: group theory, electronic structure, and lattice dynamics study. Phys Rev B 76:165124-165139
14. Bhattacharjee A, Roy D, Roy M et al (2010) Rod-like ferrites obtained through thermal degradation of a molecular ferrimagnet. J Alloys Compd 503:449-453

15. Morin FJ (1950) Magnetic susceptibility of a-Fe $\mathrm{Fe}_{2} \mathrm{O}_{3}$ and $\mathrm{a}-\mathrm{Fe}_{2} \mathrm{O}_{3}$ with added titanium. Phys Rev 78:819-820

16. Dey A, Zubko M, Kusz J et al (2019) Solventless synthesis and characterization of $a-\mathrm{Fe}, \gamma-\mathrm{Fe}$, magnetite and hematite using iron(III)citrate. Solid State Sci 95:105932-105939. https://doi. org/10.1016/j.solidstatesciences.2019.105932

17. Das B, Kusz J, Reddy VR et al (2017) Solventless synthesis, morphology, structure and magnetic properties of iron oxide nanoparticles. Solid State Sci 74:62-69

18. Lyubutin IS, Lin CR, Korzhetskiy YV et al (2009) Mössbauer spectroscopy and magnetic properties of hematite/magnetite nanocomposites. J Appl Phys 106:34311-34316

19. Diaz C, Barrientos L, Carrillo D et al (2016) Solvent-less method for efficient photocatalytic a- $\mathrm{Fe}_{2} \mathrm{O}_{3}$ nanoparticles using macromolecular polymeric precursors. New J Chem 40:6768-6776

20. Sharma G, Jeevanandam $P$ (2013) Synthesis of self-assembled prismatic iron oxide nanoparticles by a novel thermal decomposition route. RSC Adv 3:189-200

21. Han YC, Cha HG, Kim CW et al (2007) Synthesis of highly magnetized iron nanoparticles by a solventless thermal decomposition method. J Phys Chem C 111:6275-6280

22. Karimzadeh I, Dizaji HR, Aghazadeh M (2016) Preparation, characterization and PEGylation of superparamagnetic $\mathrm{Fe}_{3} \mathrm{O}_{4}$ nanoparticles from ethanol medium via cathodic electrochemical deposition (CED) method. Mater Res Express 3:95022

23. Al-Alawy AF, Al-Abodi EE, Kadhim RM (2018) Synthesis and characterization of magnetic iron oxide nanoparticles by coprecipitation method at different conditions. J Eng 24:60-72

24. Hassanjani-Roshan A, Vaezi MR, Shokuhfar A, Rajabali Z (2011) Synthesis of iron oxide nanoparticles via sonochemical method and their characterization. Particuology 9:95-99

25. Li S, Zhang T, Tang R et al (2015) Solvothermal synthesis and characterization of monodisperse superparamagnetic iron oxide nanoparticles. J Magn Magn Mater 379:226-231

26. Chin AB, Yaacob II (2007) Synthesis and characterization of magnetic iron oxide nanoparticles via w/o microemulsion and Massart's procedure. J Mater Process Technol 191:235-237

27. Takami S, Sato T, Mousavand T et al (2007) Hydrothermal synthesis of surface-modified iron oxide nanoparticles. Mater Lett 61:4769-4772

28. Zhang YC, Tang JY, Hu XY (2008) Controllable synthesis and magnetic properties of pure hematite and maghemite nanocrystals from a molecular precursor. J Alloys Compd 462:24-28

29. Bhattacharjee $A$, Roy D, Roy $M(2012)$ Thermal degradation of a molecular magnetic material: $\left\{\mathrm{N}\left(\mathrm{n}-\mathrm{C}_{4} \mathrm{H}_{9}\right)_{4}\left[\mathrm{Fe}{ }^{\mathrm{II}} \mathrm{Fe}{ }^{\mathrm{III}}\left(\mathrm{C}_{2} \mathrm{O}_{4}\right)_{3}\right]\right\}_{\infty}$. J Therm Anal Calorim 109:1423-1427

30. Dey A, Zubko M, Kusz J, Bhattacharjee A (2018) Kinetics study of the solid state reaction of iron (III) citrate leading to hematite nanoparticles. Curr Phys Chem 8:290-302

31. Bini RA, Marques RFC, Santos FJ et al (2012) Synthesis and functionalization of magnetite nanoparticles with different aminofunctional alkoxysilanes. J Magn Magn Mater 324:534-539

32. Gotić M, Musić S (2007) Mössbauer, FT-IR and FE SEM investigation of iron oxides precipitated from $\mathrm{FeSO}_{4}$ solutions. J Mol Struct 834:445-453

33. Carter N (2015) Physical properties of iron oxide nanoparticles. Honors College, Paper 210. http://digitalcommons.library.umain e.edu/honors $/ 210$

34. Tharani K, Nehru LC (2015) Synthesis and characterization of iron oxide nanoparticle by precipitation method. Int J Adv Res Phys Sci 2:47-50 
35. Basavaraja S, Balaji DS, Bedre MD et al (2011) Solvothermal synthesis and characterization of acicular $\mathrm{a}-\mathrm{Fe}_{2} \mathrm{O}_{3}$ nanoparticles. Bull Mater Sci 34:1313-1317

36. Senapati US, Sarkar D (2014) Characterization of biosynthesized zinc sulphide nanoparticles using edible mushroom Pleurotuss ostreatu. Indian J Phys 88:557-562

37. Johnson CE (1996) Characterization of magnetic materials by Mössbauer spectroscopy. J Phys D Appl Phys 29:2266-2273

38. Bhattacharjee A, Reiman S, Ksenofontov V, Gütlich P (2003) Mössbauer spectroscopy under a magnetic field to explore the low-temperature spin structure of the layered ferrimagnetic material- $\left\{\mathrm{N}\left(\mathrm{n}-\mathrm{C}_{4} \mathrm{H}_{9}\right)_{4}\left[\mathrm{Fe}^{\prime \prime} \mathrm{Fe}^{\text {III }}\left(\mathrm{C}_{2} \mathrm{O}_{4}\right)_{3}\right]\right\}_{\infty}$. J Phys Condens Matter 15:5103-5112

39. Shipilin MA, Zakharova IN, Shipilin AM, Bachurin VI (2014) Mössbauer studies of magnetite nanoparticles. J Surf Investig X-ray Synchrotron Neutron Tech 8:557-561

40. McNab TK, Fox RA, Boyle AJF (1968) Some magnetic properties of magnetite (Fe3O4) microcrystals. J Appl Phys 39:5703-5711

41. Bhattacharjee A, Rooj A, Roy M et al (2013) Solventless synthesis of hematite nanoparticles using ferrocene. J Mater Sci 48:2961-2968
42. Wignall JWG (1966) Mössbauer line broadening in trivalent iron compounds. J Chem Phys 44:2462-2467

43. Patsula V, Moskvin M, Dutz S, Horák D (2016) Size-dependent magnetic properties of iron oxide nanoparticles. J Phys Chem Solids 88:24-30. https://doi.org/10.1016/j.jpcs.2015.09.008

44. Mørup S (1990) Mössbauer effect in small particles. Hyperfine Interact 60:959-973

45. Shinjo T (1966) Mössbauer effect in antiferromagnetic fine particles. J Phys Soc Jpn 21:917-922

46. Yin $M$, Willis $A$, Redl F et al (2004) Influence of capping groups on the synthesis of $\gamma^{-} \mathrm{Fe}_{2} \mathrm{O}_{3}$ nanocrystals. J Mater Res 19:1208-1215

47. Jubb AM, Allen HC (2010) Vibrational spectroscopic characterization of hematite, maghemite, and magnetite thin films produced by vapor deposition. ACS Appl Mater Interfaces 2:2804-2812

Publisher's Note Springer Nature remains neutral with regard to jurisdictional claims in published maps and institutional affiliations. 